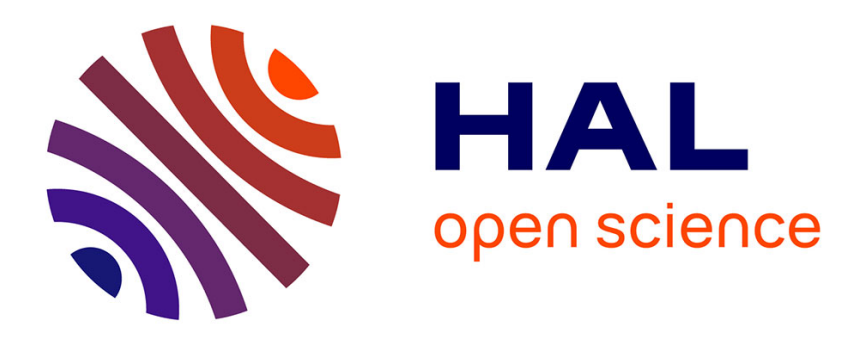

\title{
Technologies de l'implication des parents dans les institutions éducatives
}

Gilles Monceau

\section{To cite this version:}

Gilles Monceau. Technologies de l'implication des parents dans les institutions éducatives. Revue internationale de l'éducation familiale, 2010, 27 (1), 10.3917/rief.027.0017 . hal-01712958

\section{HAL Id: hal-01712958 \\ https://hal.science/hal-01712958}

Submitted on 20 Feb 2018

HAL is a multi-disciplinary open access archive for the deposit and dissemination of scientific research documents, whether they are published or not. The documents may come from teaching and research institutions in France or abroad, or from public or private research centers.
L'archive ouverte pluridisciplinaire $\mathbf{H A L}$, est destinée au dépôt et à la diffusion de documents scientifiques de niveau recherche, publiés ou non, émanant des établissements d'enseignement et de recherche français ou étrangers, des laboratoires publics ou privés. 


\section{TECHNOLOGIES DE L'IMPLICATION DES PARENTS DANS LES INSTITUTIONS ÉDUCATIVES}

\section{Gilles Monceau}

\section{L'Harmattan | « La revue internationale de l'éducation familiale »}

2010/1 n $27 \mid$ pages 17 à 35

ISSN 1279-7766

ISBN 9782296121843

\section{Article disponible en ligne à l'adresse :}

http://www.cairn.info/revue-la-revue-internationale-de-l-educationfamiliale-2010-1-page-17.htm

\section{!Pour citer cet article :}

Gilles Monceau, «Technologies de l'implication des parents dans les institutions éducatives », La revue internationale de l'éducation familiale 2010/1 ( $\left.{ }^{\circ} 27\right)$, p. 17-35.

DOI 10.3917/rief.027.0017

Distribution électronique Cairn.info pour L'Harmattan.

(C) L'Harmattan. Tous droits réservés pour tous pays.

La reproduction ou représentation de cet article, notamment par photocopie, n'est autorisée que dans les limites des conditions générales d'utilisation du site ou, le cas échéant, des conditions générales de la licence souscrite par votre établissement. Toute autre reproduction ou représentation, en tout ou partie, sous quelque forme et de quelque manière que ce soit, est interdite sauf accord préalable et écrit de l'éditeur, en dehors des cas prévus par la législation en vigueur en France. Il est précisé que son stockage dans une base de données est également interdit. 


\section{Technologies de l'implication des parents dans les institutions éducatives}

Gilles Monceau ${ }^{1}$

L'article prend appui sur deux recherches socio-cliniques en milieu éducatif menées entre 2005 et 2009 en Île de France et en Picardie (France). La première porte sur la coopération entre parents et enseignants à l'école primaire et la seconde sur les interventions d'une association d'éducation populaire dans les dispositifs d'appui à la parentalité. Le cadre politique est pris en compte, notamment à travers les rapports et circulaires produits par les ministères de l'Éducation nationale et des Affaires sociales. L'auteur avance que les dispositifs de coopération entre parents et enseignants, comme ceux d'appui à la parentalité, sont à analyser comme des technologies agissant sur l'implication des parents dans les institutions.

Mots-clés : coopération, implication, institution scolaire, travail social, parentalité.

\section{Introduction}

Depuis le début des années 2000, une politique visant les parents s'affirme en France à la fois dans et hors de l'institution scolaire. À l'école, elle met en avant l'objectif de donner un rôle et une place aux parents d'élèves. Hors l'école, elle se présente davantage comme venant appuyer et accompagner les parents en difficulté.

${ }^{1}$ Maître de conférences, HDR, CIRCEFT, Université Paris 8 Vincennes-SaintDenis, 2, rue de la Liberté, 93526 Saint-Denis cedex 02 (France).

Contact : gilles.monceau@univ-paris8.fr

La revue internationale de l'éducation familiale, n²7, 2010 
Dans les deux cas, le mot " assistance » est évité au profit de ceux d' « autonomie » et de « responsabilité ». La volonté politique de donner des droits nouveaux aux parents s'accompagne indissociablement de celle de les rendre capables d'assumer leurs responsabilités. L'analyse formulée par Robert Castel à propos de l'émergence, depuis la Révolution française (Castel, 2009), de l'individu moderne sous la figure du citoyen constitue une toile de fond sur laquelle nous pouvons examiner la manière dont l'État traite aujourd'hui les citoyens-parents. Les droits concédés aux individus sont justifiés par les compétences qu'ils doivent acquérir et se paient par un élargissement de leurs responsabilités, y compris pénales ${ }^{1}$.

Les travaux que nous allons présenter ici nous conduisent à avancer qu'au-delà des objectifs affirmés d'aide et de soutien aux parents, les dispositifs de coopération parents/enseignants et ceux d'appui à la parentalité sont d'abord à analyser comme des technologies ${ }^{2}$ agissant sur l'implication des parents dans les institutions.

\section{L'individualisation de la responsabilité parentale à l'école}

Dominique Glasman avait attiré l'attention des chercheurs sur les usages respectifs des termes "parents " et "familles », en particulier dans les zones d'éducation prioritaire ${ }^{3}$ (Glasman, 1992). À partir de quelques enquêtes de terrain, il remarquait que les professionnels utilisaient davantage le second terme en ZEP alors que le premier était plus spontanément utilisé dans d'autres quartiers. Quinze années plus tard, le mot "parent» tend à être partout utilisé comme les textes ministériels y incitent fortement. La notion de famille semble désormais bien trop imprécise pour pouvoir y adosser une politique.

\footnotetext{
${ }^{1}$ Des sanctions pénales peuvent intervenir en application de l'article 227-17 du code pénal qui dispose que : « Le fait, par le père ou la mère légitime, naturel ou adoptif, de se soustraire, sans motif légitime, à ses obligations légales au point de compromettre gravement la santé, la sécurité, la moralité ou l'éducation de son enfant mineur est puni de deux ans d'emprisonnement et de $200000 \mathrm{~F}$ d'amende. ». Cet article a été inséré dans le code pénal par la loi n 98-1165 du 18 décembre 1998.

${ }^{2}$ Cette notion de technologie renvoie aux travaux de Michel Foucault concernant la notion de « technologie politique » (Foucault, 1978).

${ }^{3}$ Les zones d'éducation prioritaires (ZEP) ont été instaurées en France en 1982 avec l'objectif de concentrer des moyens humains et financiers sur des établissements scolaires d'aires géographiques jugées socialement et culturellement défavorisées.
} 
Selon qu'ils sont individus "par défaut » ou " par excès » (Castel, 2009), les parents n’investissent pas de la même manière la réglementation et les dispositifs mis en œuvre pour les accompagner dans la tenue de leurs (nouveaux) rôles ${ }^{1}$. Dans tous les cas, il y a individualisation de la responsabilité, même lorsqu'il existe des couples parentaux ou des droits collectifs. Ainsi, la circulaire du 31 aout 2006 du ministère de l'Éducation nationale précise que : «C’est au niveau local de l'école ou de l'établissement scolaire que doit se mettre en place un dialogue confiant et efficace avec chacun des parents d'élèves» ${ }^{2}$. Les chefs d'établissements ou les enseignants ne peuvent donc pas se contenter de dialoguer avec les représentants des parents de leurs élèves ni même avec seulement l'un des parents de chaque élève. La même circulaire indique d'ailleurs que: «Compte tenu de l'évolution sociologique des familles, il est aujourd'hui nécessaire de considérer que l'institution peut avoir affaire à deux interlocuteurs pour un élève, le père et la mère. En effet, conformément aux dispositions de l'article 371-1 du code civil, l'autorité parentale est en principe exercée conjointement par les père et mère, quelle que soit leur situation (mariés ou non, séparés, divorcés...). Les écoles et établissements scolaires doivent pouvoir entretenir avec les deux parents les relations nécessaires au suivi de la scolarité de leurs enfants ».

Les droits collectifs accordés aux associations de parents d'élèves, principalement en matière d'information, ne se substituent donc pas aux droits individuels de chaque parent. Le responsable de l'établissement doit donc être attentif à la fois aux demandes formulées collectivement et à celles qui sont plus individuelles. La circulaire indique que : «Les directeurs d'école ou les chefs d'établissement, les enseignants et l'ensemble des personnels de l'établissement veilleront à être à l'écoute des attentes des parents. Les demandes individuelles d'information ou d'entrevue devront recevoir une réponse ».

Cette recommandation peut être vue comme une charge particulièrement importante pour les chefs d'établissements mais elle légitime également le fait qu'ils puissent s'adresser directement à chaque parent sans passer par l'intermédiaire des associations et des représentants élus. Peut-être s'agit-il de corriger l'effet, souvent observé,

${ }^{1}$ Les premiers ne satisfont pas aux attentes d'autonomie et de responsabilité qui sont formulées envers eux alors que les seconds les dépassent en les intégrant à leurs propres stratégies.

2 « Le rôle et la place des parents à l'école », circulaire nº 2006-137 du 25-8-2006 parue au Bulletin officiel de l’Éducation nationale n³1 du 31 août 2006.

La revue internationale de l'éducation familiale, $\mathrm{n}^{\circ} 27,2010$ 
d'un éloignement de la majorité des parents d'élèves lorsqu'une minorité active d'entre eux entretient des rapports privilégiés avec les enseignants (van Zanten, 2001)?

\section{La politisation de la notion de parentalité}

La notion de parentalité apparaît d'abord en psychologie clinique à la fin des années 1950 pour désigner le désir d'enfant et les remaniements psychiques qui accompagnent sa naissance. C'est encore dans cette logique que Sylvain Missonnier parle d'une "relation d'objet virtuel » dans une publication de 2004 (Missonnier, 2004). Ainsi comprise, la notion ne concerne donc pas nécessairement tous les parents ni seulement les parents biologiques, c'est un processus que le sujet développe dès l'enfance et qui engage des évolutions psychiques.

La notion est donc très différente de celle de parenté, plus particulièrement travaillée en anthropologie pour désigner d'abord l'inscription de l'individu dans une structure familiale, dans une lignée et donc dans une culture singulière donnant un sens spécifique au fait d'être ou de devenir père ou mère. Dans ce second cadre, le désir d'enfant comme la manière d'être parent sont alors d'abord situés dans des modèles hérités.

La notion de parentalité diffuse de la psychologie clinique vers le travail social dans les années 1990. Ainsi le Dictionnaire critique de l'action sociale la mentionne dans son édition de 1995 comme étant un terme du vocabulaire médico-psycho-social désignant principalement la fonction parentale, support de l'évolution psycho-affective de l'enfant, et intègre les responsabilités morales qui s'y rapportent (Barreyre \& Bouquet, 1995).

Enfin, à la fin des années 1990, la notion va devenir pleinement politique avec la mise en œuvre des Réseaux d'Écoute, d'Appui et d'Accompagnement des Parents (REAAP) en 1999, ce dispositif ayant pour principal objectif de susciter et de fédérer à l'échelle nationale des initiatives diversifiées (d’organismes publics et privés, de professionnels et de bénévoles) en direction des parents les plus en difficulté. Il faut se souvenir que les années 1990 sont aussi celles de la montée d'une préoccupation médiatique et politique pour la violence en milieu scolaire ainsi que du thème du parent démissionnaire.

Le rapport du groupe de travail dirigé par le psychiatre psychanalyste Didier Houzel (Houzel, 1999) va contribuer à faire passer 
la parentalité de la clinique à la sphère politique. En s'appuyant sur une dizaine de cas, il va produire une nouvelle approche de la notion de parentalité qui décline trois axes (exercice du droit, expérience subjective et pratique effective). La mise en œuvre d'une action politique sera facilitée par les recommandations émises par le rapport.

Cette conceptualisation, comme les propositions qui en découlent, sont donc élaborées à partir de l'examen de situations de "défaillances » de la fonction parentale. C'est ce qu'explique Catherine Sellenet qui a elle-même participé à ce groupe de travail (Sellenet, 2007). D’autres observateurs avancent, comme Claire Neirinck, que c'est l'accent mis sur les compétences parentales (donc également sur leurs incompétences) qui explique le passage de la parenté à la parentalité dans les discours de politique familiale (Neirinck, 2002).

Enfin, le succès de la notion de parentalité doit aussi, selon C. Sellenet (Sellenet, 2007) et D. Fablet (Fablet, 2008), au fait qu'elle a offert aux professionnels de l'enfance, dès le début des années 1980, une possibilité de reconsidérer l'objet même de leur pratique. En passant d'une centration sur l'enfant (réduisant au maximum les interactions avec les parents) à une aide à apporter aux parents pour qu'ils soient en mesure d'élever et d'éduquer leurs enfants, la posture professionnelle prenait une ampleur nouvelle. Cette évolution n'est d'ailleurs pas sans conséquence pour l'enfant qui passe alors au second plan. Sellenet utilise d'ailleurs le terme de «parentalisme » pour décrire cette attention nouvelle portée au parent plutôt qu’à l'enfant.

Il ne faut sans doute pas voir dans cette évolution la fin de la " passion de l'enfant » que pointait Laurence Gavarini mais plutôt son amplification (Gavarini, 2001). En effet, l'enfant paraît aujourd'hui davantage encore réduit à un statut d'objet (de désir, d'attention, de conflit, de projet...). En prenant les parents pour cibles, l'action publique affiche la volonté de restaurer leur place, comme en contrepoint de la célébration des droits et de la parole de l'enfant dans les dix années précédentes. Dans le même temps, les défaillances parentales font l'objet d'une plus grande attention. Les parents doivent désormais pouvoir être écoutés et accompagnés au sein de dispositifs dont le sociologue Didier Fassin montre qu'ils caractérisent, depuis le début des années 1990, un nouveau mode de gestion des problèmes sociaux (Fassin, 2004). Que les difficultés des individus concernent leurs situations économique, familiale ou sanitaire, la tendance serait à la dépolitisation par le renvoi de chacun à sa problématique personnelle. 


\section{Implications parentales}

Le concept d'implication est défini ici en référence aux travaux de René Lourau (Lamihi \& Monceau, 2002), l'implication parentale est donc l'ensemble des relations que les parents entretiennent avec les institutions. L'implication n'est donc pas à confondre avec l'investissement ou engagement. Les parents d'enfants scolarisés sont impliqués dans l'institution scolaire même s'ils ne s'investissent pas dans le fonctionnement de l'établissement scolaire ni ne s'engagent dans une association de parents. L’implication n'est donc pas nécessairement volontaire (la scolarisation étant obligatoire ${ }^{1}$ ) ni même consciente. Des parents d'élèves qui ne se rendent jamais physiquement dans l'école de leurs enfants n'en sont pas moins impliqués scolairement, qu'ils le veuillent ou non. Ils entretiennent des relations (même si elles sont difficiles) avec l'institution scolaire (Monceau, 2008 ${ }^{\mathrm{a}}$ ). Ces implications peuvent se décliner sur les plans affectif, organisationnel ou idéologique.

Si des politiques sont arrêtées et mises en œuvre pour donner un rôle et une place aux parents dans l'institution scolaire, comme pour appuyer et accompagner ceux dont la parentalité serait défaillante, c'est peut-être qu'il ne s’agit pas seulement de répondre à des attentes ou des besoins des parents-citoyens mais que les institutions ont elles-mêmes besoin d'impliquer les parents.

\section{Deux recherches socio-cliniques en milieu éducatif}

L'article repose sur les résultats de deux travaux socio-cliniques : une enquête-participation menée en 2005-2006 à propos de la coopération parents/enseignants et une recherche-action menée en 20082009 sur l'intervention de l'Éducation populaire ${ }^{2}$ dans les dispositifs d'appui à la parentalité. Les deux commandes (la première de la Fondation de France et la seconde des Centres d'Entraînement aux Méthodes d'Education Active - CEMEA) renvoient à deux aspects d'un même enjeu politique que nous pourrions formuler ainsi : comment faire en sorte que les parents endossent pleinement la responsabilité de

\footnotetext{
${ }^{1}$ En France, l'instruction est obligatoire (contrôlée par le ministère de l'Éducation nationale) mais non la scolarisation. Cependant, pour les parents visés par la politique de la parentalité, il est quasiment impossible d'instruire leurs enfants sans les scolariser.

2 Le mouvement de l'Éducation populaire regroupe, en France, un ensemble d’organismes associatifs qui mènent des actions éducatives (en direction des enfants et des adultes), en dehors des structures étatiques mais souvent en partenariat avec celles-ci.
} 
l'éducation de leurs enfants sous un mode institutionnellement acceptable?

En permettant des investigations à l'intérieur et à l'extérieur de l'école, les deux chantiers précédemment évoqués ont permis d'observer la déclinaison de cette politique de la parentalité dans deux cadres institutionnels différents. Une même orientation politique y est explicitement affirmée : restaurer et soutenir la fonction parentale et les responsabilités qui s'y attachent.

La démarche de recherche adoptée, que je qualifie de socio-clinique institutionnelle, prend appui sur des commandes auxquelles il est répondu sous différentes modalités (analyse institutionnelle des pratiques, recherche-action, intervention socio-analytique ou enquête-participation) (Monceau, 2003). Les chercheurs travaillent au plus près des sujets (ici des parents et des professionnels de l'éducation) concernés par la problématique de recherche (ici la politique de la parentalité) sur un temps relativement long (une année et demie dans les deux cas) et en étant attentif au contexte institutionnel dans lequel se déroule la recherche (discours politiques, lois, règlements, rapports ministériels), ainsi qu'aux demandes des interlocuteurs de terrain (demandes d'analyse de leurs propres pratiques et demandes de pouvoir élaborer et exprimer leurs propres positions le plus souvent).

Cette démarche use principalement de techniques telles que les entretiens individuels et collectifs, l'observation participante et l'analyse documentaire. Les entretiens collectifs donnent particulièrement l'opportunité de restituer, aux sujets, des éléments issus des premières investigations. Ce faisant, les enjeux sociaux apparaissent alors de manière plus manifeste dans la dynamique des échanges entre les participants. Les enregistrements audio de tous les entretiens sont transcrits. Ils constituent, avec les notes d'observation et les documents collectés, un corpus faisant l’objet d'analyses différées.

\section{Une recherche sur la coopération parents/enseignants}

La première investigation répond à une commande de la Fondation de France ${ }^{1}$ émise dans le cadre de son programme " Enfance ». Cette recherche collective a donné lieu à la publication d'un ouvrage (Kherroubi, 2008). La commande portait sur les conditions de la

\footnotetext{
${ }^{1}$ La Fondation de France est un organisme de statut privé qui finance des actions en faveur du lien social. Elle fonctionne grâce à des dons et legs privés.
} 
coopération parents/enseignants à partir de l'analyse de pratiques de coopération déjà mises en oeuvre en écoles primaires. Les résultats utilisés ici concernent sept écoles ayant fait l'objet d'un travail qui a combiné des observations directes de différentes situations (entrée et sortie des enfants, classe, récréation, ateliers, réunions entre enseignants, entre enseignants et parents), la consultation des projets écrits des écoles et la réalisation d'entretiens individuels et collectifs ${ }^{1}$.

Cinq de ces écoles avaient reçu un prix de la Fondation pour leur action de coopération avec les parents. Six étaient classées ZEP, mais ceci était susceptible d'être remis en cause pour deux d'entre elles, compte tenu des évolutions foncières et démographiques des quartiers concernés. Ces écoles ZEP (aux publics souvent hétérogènes) étaient situées en zone urbaine (à Paris, en banlieue parisienne et dans une ville moyenne), l'école hors ZEP se trouvait en milieu semi-rural et recevait une population d'enfants d'employés, d'ouvriers et de cadres moyens.

Durant la même période (2005-2006), l’inspection générale de l'Éducation nationale menait une enquête portant sur la place des parents à l'école (Warzée, 2006). La circulaire produite par le ministère de l'Éducation nationale, sur la base de ce travail, porte sur le fonctionnement interne de l'école, les droits des parents à l'information et les modalités de leur participation aux instances réglementaires. Elle indique qu'un local peut être mis à la disposition des représentants de parents d'élèves non affiliés, de manière temporaire ou permanente, dans les mêmes conditions que pour les associations de parents d'élèves.

Le rapport Warzée avait cependant un champ plus large que celui de la circulaire et mettait en relation l'intra et l'extra-scolaire. Il établissait un inventaire des dispositifs d'aide à la parentalité destinés aux «parents les plus démunis ». La liste des dispositifs cités est sans ambiguïté sur ce point: centres de ressources enfance, famille, école (CREFE) de la région Rhône-Alpes ; contrats locaux d'accompagnement à la scolarité (CLAS) financés par la Caisse d'allocations familiales et ciblant en priorité les élèves relevant des réseaux d'éducation prioritaire (REP) et des zones urbaines sensibles (ZUS); réseaux d'écoute, d'appui et d'accompagnement des parents (REAAP); jeu de fiches pratiques à l'intention des familles éditées par la délégation interministérielle à la famille; contrats éducatifs locaux (CEL). Enfin, le rapport cite «l'initiative prise par la Fondation de France ».

1 Les investigations de terrain ont été menées par Brigitte Larguèze, Ana Athanasopoulou, Maria-Renata Prado et moi-même. 
Constatant que les équipes enseignantes se plaignent de leur difficulté à entrer en relation avec certains parents et que cette difficulté concerne aussi les associations de parents d'élèves, le rapport propose de : «mettre en place, à destination des parents qui ne sont pas ou peu représentés dans les grandes fédérations, des « Ateliers » ou «Écoles des parents », situés en dehors de l'établissement ou de l'école (local municipal, associatif, CIO...), dans lesquels seraient expliqués aux parents qui le souhaitent le fonctionnement de l'école, les attentes de l'institution, leurs droits, les procédures d'orientation, etc. Ces ateliers pourraient être animés et co-gérés par les fédérations de parents d'élèves, les établissements et personnels de l'éducation nationale (et en particulier, les personnels d'orientation), les associations, etc. » (Warzée, 2006, p. 63). Cette proposition s’inscrit dans la logique de la politique des REAAP précédemment évoquée.

Dans un paragraphe de sa conclusion, le rapport formule une évaluation de la relation école/famille: «La tension école/famille, souvent perceptible, est donc assez normale. Elle est dynamique et féconde si elle reste contenue dans de justes limites. Le réglage est cependant toujours délicat (...). Est-il nécessaire de rappeler que la légitime reconnaissance des droits des parents d'élèves ne doit pas pour autant aboutir à compliquer la mission déjà difficile des personnels chargés de l'enseignement des élèves ? »(Warzée, 2006, p. 62). Les enquêteurs, s’ils sont préoccupés par la relation trop distendue entre l'École et les "parents les plus démunis », le sont aussi par le risque de voir des parents plus mobilisés compliquer la tâche des enseignants en usant des droits nouveaux.

Les politiques familiale et scolaire actuelles visent à redéfinir les rapports entre services publics et usagers au moins autant qu'à intervenir sur l'éducation des enfants, ce qui passe par une intervention sur la subjectivité des individus (Genard \& Cantelli, 2007). Réputé autonome et responsable, le parent doit assurer le pilotage de la scolarité de son (ses) enfant(s) et ceci quel que soit son niveau d'information sur les rouages institutionnels de l’École. Si l’Inspection générale signale le risque qu’il y aurait à compliquer la tâche des enseignants, ce souci n'est pas nécessairement relayé par les responsables politiques qui peuvent voir dans cette intervention des parents un bon moyen d'accélérer certaines réformes.

Cette politique accompagne les revendications des associations de parents d'élèves dont on sait qu'elles regroupent les parents les mieux 
informés et les plus à même de tirer avantage du processus. Comme Martine Barthélémy l'a montré dans son enquête sur les trois principales organisations françaises de parents d'élèves (FCPE, PEEP et UNAPEL), les parents les plus mobilisés peuvent cependant combiner cette sorte de consumérisme avec un engagement militant au service de l'intérêt général (Barthélémy, 1995). Nous avons d'ailleurs pu l’observer nousmêmes lors de nos enquêtes. Les histoires de ces associations nationales font aussi apparaître leurs différents rapports à l'École, à la fois pragmatiques et politiques (Gombert, 2006).

Lors des entretiens que nous avons menés avec les directeurs d'écoles et les enseignants, il est ainsi apparu que les modalités d'évaluation des projets d'école incitent des directeurs à « coopter » des parents lorsque les sièges au conseil d'école ne sont pas pourvus spontanément. Une augmentation du taux de participation à l'élection et celle du nombre de sièges pourvus sont en effet des indicateurs valorisants. Les directeurs débordent alors leur rôle de garants de l'application des textes réglementaires pour agir directement sur la production d'un taux de participation qui entrera positivement en compte dans l'évaluation de leur propre action (Monceau, 2009b).

Que le directeur d'école coopte lui-même des candidats à l'élection des représentants de parents n'est guère conforme au principe d'une représentation autonome des parents. En faisant ainsi, il choisit de fait ses interlocuteurs. Une finalité nouvelle est alors donnée à ce dispositif de représentation. Le conseil d'école devient un outil de formation et de mobilisation de parents initialement en retrait, une technologie pour agir sur leur implication dans l'institution scolaire. Certains parents rencontrés durant les enquêtes de terrain expliquent en détail ce qu’ils ont découvert en fréquentant cette instance à laquelle ils ne se seraient pas autorisés d'eux-mêmes à se porter candidats. Cette dynamique est inverse à ce que d'autres travaux montrent, dans le cas de parents de classes moyennes en particulier, d'un investissement dans l'école qui prolonge des engagements antérieurs (associatif, politique ou syndical) 1. Cependant, certains parents ainsi sollicités pour être candidats disent, après quelques mois, leur déception d'avoir découvert qu'il n'était pas question de pédagogie dans les réunions du conseil d’école. Ils préfèrent alors ne pas se représenter l'année suivante pour privilégier des activités qui leur permettent d'entrer dans la classe.

1 Ceux, déjà cités, de Martine Barthélémy en particulier. 
Les observations du fonctionnement ordinaire de trois des sept écoles primaires qui ont constitué notre terrain de recherche ont montré que les enseignants motivent d'abord la coopération par la volonté de rapprocher de l'école les parents les plus difficiles à rencontrer. Lors des entretiens individuels, ils disent couramment qu'ils cherchent « des trucs pour faire venir les parents à l'école ».

Dans certrains cas, la coopération permet à des directeurs d’être moins exposés à des conflits avec les parents les plus mobilisés. Plusieurs d'entre eux nous ont ainsi expliqué comment la diversification des modes de participation à la vie de l'école (animation d'atelier, accompagnement des classes en sortie, apport de petit matériel) leur avait permis d'augmenter le nombre de leurs interlocuteurs parentaux et de minorer ainsi la place des représentants élus. Avant que d'être un moyen de faciliter les apprentissage scolaires via une meilleure communication avec les parents, la coopération est un outil de pilotage de l'établissement sur le plan de ses rapports à son public.

\section{Une recherche-action portant sur l'appui à la parentalité}

Les CEMEA ${ }^{1}$ sont sollicités par des structures (centres sociaux, mais aussi collèges et associations de parents) pour aider à la mise en œuvre d'actions susceptibles de s'inscrire dans la politique de la parentalité. Dans un second temps, ils sollicitent des chercheurs pour analyser les enjeux de leurs propres interventions de terrain dans cette politique. Cet emboîtement des commandes n'est pas anodin, il illustre un mode de gestion qui tend à externaliser certaines actions de l'État.

La commande que nous recevons des CEMEA trouve son origine dans l'émergence d'un secteur d'activité dont la circulaire du 13 juillet 2004, relative aux « Réseaux d'Écoute, d'Appui et d'Accompagnement des Parents (REAAP) », a fixé le cadre : "En élevant leurs enfants, les parents développent des compétences ; ils connaissent aussi un jour ou l'autre des doutes. Par l'échange, le dialogue et l'écoute, les parents peuvent s'aider mutuellement à retrouver des repères ou à mieux trouver des réponses à leurs interrogations (...). Redonner confiance aux parents et les aider à assurer leur rôle parental est l'un des objectifs de la

1 Les centres d'entraînement aux méthodes d'éducation active (CEMEA) appartiennent au mouvement de l'Éducation populaire. Ils développent des actions d'animation culturelle et de formation (en particulier formation d'animateurs de centres de vacances) et travaillent fréquemment en partenariat avec les établissements scolaires. 
politique familiale qui repose en premier lieu sur l'intérêt supérieur de l'enfant. Accompagner les parents dans leur rôle de parents est donc une priorité ».

Cet extrait pose les objectifs de la politique familiale gouvernementale. Il exprime, aussi une première limite, voire une contradiction. Si le texte vise à la constitution de réseaux permettant aux parents qui éprouvent «des doutes » de pouvoir s'entraider, il s'agit aussi, et peut-être surtout, de les accompagner «dans leur rôle de parents ». Cette dernière phrase suggère des interventions spécialisées qui permettront à cette politique de s’actualiser.

La circulaire s'accompagne d'une charte qui fixe dix "principes d'action et d'animation " dont les deux premiers sont de "valoriser prioritairement les rôles et les compétences des parents » et de «favoriser la relation entre les parents ». Les huit principes suivants s'adressent directement aux « responsables des lieux et structures fréquentées par les parents » et au type de fonctionnement qu'ils doivent mettre en œuvre pour assurer l'ouverture, l'efficacité et la pérennité du dispositif.

Ces principes définissent la stratégie adoptée pour mobiliser les parents « en doute » en "valorisant leurs compétences ». Elle prévoit de mobiliser et de former des intervenants qui inscrivent, dans la durée, cette action en cohérence avec leurs autres actions. Le texte ne se présente pas comme voulant imposer de bonnes pratiques aux parents en difficulté ${ }^{1}$ mais prévoit de faire en sorte que ceux-ci soient accompagnés par des actions de proximité (non nécessairement pilotées par des professionnels).

Dès 2004, un bilan de l'action des REAAP a été tiré par l'Inspection Générale des Affaires Sociales sous le titre : Evaluation du dispositif des Réseaux d'écoute, d'appui et d'accompagnement des Parents. Ce rapport souligne que le coût du dispositif est faible pour l'État et qu'il n'a pas augmenté malgré un nombre croissant d'initiatives de terrain. L’inspection générale remarque en conséquence la forte "productivité " du dispositif. Au plan plus qualitatif, les études de cas menées par les enquêteurs font surtout apparaître des « changements de regard », tant des

${ }^{1}$ Lorsqu'il est question de «bonnes pratiques ", c'est à propos de l'action des professionnels et bénévoles impliqués dans l'organisation des actions. Cf. le Guide de bonnes pratiques de soutien à la parentalité publié en 2008 par la Fédération nationale des associations d'accueil et de réinsertion sociale et la Caisse nationale d'allocations familiales. 
parents que des professionnels, et des effets de «dissémination ». La mission d'enquête préconise, entre autres recommandations, que l'évaluation du dispositif intègre des " enquêtes de satisfaction " auprès des parents. Cette proposition marque bien sûr une rupture avec les pratiques du travail social.

D’autres bilans, plus locaux, pointent la difficulté de mettre en œuvre un partenariat effectif avec les parents dans l'organisation et l'animation de ces actions. Ce sont les professionnels, voire des militants bénévoles dans le cas de certaines associations, qui conservent l'initiative voire qui trouvent dans cette politique de nouveaux moyens d'action.

La recherche-action pour laquelle nous avons été sollicités dans ce cadre $^{1}$ a articulé des temps d'analyse collective des pratiques, des entretiens individuels et collectifs (formels et informels) avec quatorze personnes impliquées, des observations en situation sur quatre sites, les rédactions de quatre monographies traitant de cinq actions, la tenue de cinq séances de travail avec le groupe des sept militants (bénévoles et salariés) concernés par les actions et de deux séances de restitution élargies à leurs partenaires commanditaires et professionnels de terrain ainsi que le dépouillement de la principale revue des CEMEA de 1946 à $2009^{2}$. L'action des militants alimente la recherche qui, en retour, génère des réaménagements de celle-ci.

Parmi les actions concernées par la recherche-action, celles qui se rangent sous l'intitulé "cafés de parents » sont sans doute les plus connues. Elles prennent souvent la forme de groupes de parole dans lesquels des parents, qui sont quasi exclusivement des mères, viennent échanger sur leur manière d'éduquer leurs enfants. Il arrive que des pères participent aussi, mais de manière plus ponctuelle.

D’autres actions prennent la forme d'ateliers ou de soirées jeux. Un temps et un espace sont aménagés dans lesquels les parents viennent jouer avec leurs enfants à l'intérieur de la structure (centre social ou établissement scolaire).

${ }^{1}$ La recherche-action financée par l'Association nationale des CEMEA a été menée avec l'Association territoriale de Picardie (région du nord de la France qui regroupe trois départements), sur des dispositifs que celle-ci anime.

2 Silvia Tedesco, Céline Georges et Aurélie Maurin ont participé à différents temps de ce travail. Elles ont fait part de leurs observations lors de séances de travail entre chercheurs, hors du terrain. 
Un troisième type d'action est la formation de délégués de parents (et plus largement des parents d'élèves). Ce domaine est plus ancien que la politique "parentalité ». Au début de la recherche-action, nous n'avions pas intégré ces formations à notre corpus, mais elles se sont progressivement imposées comme mobilisant la même approche de la part des militants CEMEA. Ce sont d'ailleurs bien souvent les mêmes qui interviennent dans ces différentes actions.

Enfin, il faut citer des formations à destination des parents portant sur l'outil informatique et l'usage d'internet. Loin d'être seulement techniques, ces formations sont des espaces où des parents viennent exprimer leurs inquiétudes et leurs difficultés concernant la manière dont l'informatique et internet viennent déranger l'éducation familiale. Dans certaines familles, la réduction de la fracture numérique a fragilisé.

Toutes les rencontres et entretiens formels ont été enregistrés et transcrits. En menant le travail sur une seule région, nous avions la possibilité de mieux contextualiser l'environnement social et institutionnel. Par ailleurs, le fait de croiser différentes techniques (observations, entretiens, écriture de monographie, analyse de documents) a permis de donner plus de relief à nos résultats. Les déplacements sur les terrains et l'organisation de séances de restitution avec des personnes extérieures à l'association ont permis de tester les analyses produites à mesure qu'elles étaient formulées.

Comme dans le cas de la coopération parents/enseignants, notre démarche combine l'analyse des pratiques professionnelles (ou bénévoles/militantes) et des évolutions institutionnelles qui en constituent le contexte. Ainsi, on ne considèrera pas qu'il y a rapport de cause à effet entre ces deux dimensions. Elles n'expriment pas non plus deux niveaux différents qui se superposeraient l'un à l'autre. En effet, l'institution imprègne les pratiques aussi bien que celles-ci actualisent l'institution et rétroagissent sur elle (Monceau, 2008 ${ }^{\mathrm{b}}$ ).

La demande récurrente qui a émergé à mesure qu’avançait le travail, a concerné la difficulté des établissements ou associations à toucher leurs publics. Sous des modulations variées, est revenue la nécessité (qui est aussi une injonction gouvernementale) d'intensifier les relations avec les parents d'élèves, les usagers, les habitants. L’idée est partout présente, sous le mode de l'évidence, qu'une relation plus régulière avec les parents permet de travailler plus efficacement avec les enfants. En conséquence, lorsque les parents d'un enfant en difficulté (sociale, psychologique ou scolaire) entretiennent peu de relations avec 
l'établissement, leur responsabilité (voire leur culpabilité) dans celle-ci se trouve confirmée aux yeux des professionnels ou bénévoles. Leurs propos sont tout à fait convergents sur ce point : «les parents doivent donner l'exemple à leur enfant en participant à nos actions », «les parents ne peuvent pas aider leur enfant s'ils ne viennent pas nous rencontrer ».

Remarquons que dans l'histoire des institutions éducatives en générale et de l'institution scolaire en particulier, cette évidence est un fait relativement nouveau. L’École républicaine reposait plutôt sur le principe de la séparation entre l'éducation appartenant aux parents et l'instruction relevant des enseignants.

\section{Discussion : impliquer les parents}

Durant la recherche-action, nous avons constaté que les demandes adressées aux CEMEA par les responsables de collèges, de centres sociaux ou d'associations de parents étaient structurées par une volonté de nouer ou de renouer des relations suivies avec les usagers : parents d'élèves de l'établissement ou parents du quartier. Si les commandes explicites concernent bien l'appui à apporter aux parents, les demandes plus implicites renvoient davantage à une médiation à effectuer afin de créer ou de recréer du lien social. Ce constat rejoint celui qui avait pu être fait lors de la recherche portant sur les dispositifs de coopération parents/enseignants.

Tout se passe comme si les dispositifs, de coopération dans l'enceinte scolaire ou d'appui à la parentalité en milieux scolaire et non scolaire, avaient pour principal objectif d'atteindre un public insuffisamment présent, réactif et interactif. Favoriser la scolarité de l'enfant ou soutenir la place du parent semble alors secondaire ou plutôt soumis au préalable d'un rapprochement des parents avec les institutions. Il s'agit prioritairement, pour les professionnels, d'inventer des dispositifs permettant d'agir sur les implications parentales (leurs rapports aux institutions). Pour que les institutions et les professionnels qui les incarnent puissent accomplir leur mandat social, pour que les usagers puissent continuer à croire en eux, il est nécessaire de renforcer le lien entre sujets et institutions. Ce renforcement passe par la mise en œuvre de dispositifs générateurs d'implication mais pas nécessairement d'engagement.

Les observations précédentes peuvent donner à penser que les institutions contemporaines sont en difficulté, qu'elles ne parviendraient plus à agir sur les individus. Ceci n'est qu'une représentation parmi 
d'autres. Il est aussi possible d'y voir une extension continue de l'influence des institutions. Ces dernières sont moins identifiables qu'hier à des organisations aux contours clairement délimités, les rapports hiérarchiques et les responsabilités y sont moins lisibles. Ces évolutions instituent des rapports différents aux institutions, de nouvelles manières d'y être impliqué. Les phénomènes d'externalisation diminuent le poids des infrastructures organisationnelles en même temps qu'ils étendent les ramifications institutionnelles bien au-delà de l'organisation. Ainsi, de nombreuses associations émargent aux crédits générés par la politique "parentalité », quels que soient leurs positionnements idéologiques propres, elles contribuent à élargir le rayon d'action des politiques publiques en tentant d'impliquer les parents au-delà des limites des institutions traditionnelles.

Catherine Sellenet consacre un passage de son ouvrage (Sellenet, 2007) à l'apparition du terme "implication » dans les recherches en sciences de l'éducation consacrées à l'éducation familiale. Remarquant que la notion est utilisée en psychologie et sociologie du travail, elle avance que sa diffusion indiquerait le développement d'une logique d'entreprise au sein de la famille. Il faut cependant signaler l'usage, bien plus ancien en sciences de l'éducation, d'un concept d'implication dans le domaine de l'analyse institutionnelle ${ }^{1}$. En focalisant l'analyse sur la nature de l'implication plutôt que sur sa mesure, le concept vise à élargir l'analyse en situant les actes et pensées des individus dans des évolutions institutionnelles qui les dépassent.

Étudier les dispositifs de coopération avec les parents dans le champ scolaire ou d'appui de leur parentalité dans celui de l'action sociale comme relevant d'une technologie de l'implication (institutionnelle) conduit également à reconsidérer le discours désormais banalisé sur le déclin des institutions (Dubet, 2002). Comme le remarque Castel (Castel, 2009) avec d'autres auteurs, les individus les plus en difficulté (Le Strat, 1997) et plus largement en situation de demande (Samson, 2005) doivent faire la preuve de leur investissement dans un projet pour eux-mêmes, voire un projet d'eux-mêmes. Ils endossent ainsi les attributs d'individus autonomes, responsables et sont donc potentiellement coupables en cas d'échec.

${ }^{1}$ Et plus particulièrement dans les travaux de Lourau (Lamihi \& Monceau, 2002) comme indiqué plus haut. 
Les parents «par défaut ${ }^{1}$, toujours trop peu investis aux yeux des professionnels, ne les inquiètent cependant pas nécessairement davantage que les " parents par excès » qui surinvestiraient trop l'éducation de leurs enfants en leur faisant porter le poids de leurs propres aspirations. Dans les dispositifs étudiés, les premiers sont considérés avec davantage de bienveillance, peut-être parce qu'ils sont plus gratifiants pour les professionnels. Les seconds assument leurs responsabilités parentales en usant des opportunités institutionnelles nouvelles pour poursuivre leurs objectifs. Ce faisant ils sont aussi plus rétifs aux avis et à l'action des professionnels.

La réalité quotidienne des relations entre parents et institutions est bien sûr plus composite que cette opposition binaire entre deux types de parents. Malgré des ressources fort différentes, le refus d'être objectivé par les professionnels est commun à tous les parents que nous avons rencontrés, bien que se manifestant de manières très variées (Monceau, $2009^{\mathrm{a}}$ ). L'analyse socio-clinique de ces résistances, toujours contradictoires, est à poursuivre si l'on veut mieux saisir comment évoluent nos institutions. Il en va des technologies de l'implication comme des autres, elles ne fonctionnent pas nécessairement de la manière prévue.

\section{Références bibliographiques}

Barreyre, J-Y., \& Bouquet, B. (Eds.) (1995). Dictionnaire critique de l'action sociale. Paris : Dalloz.

Barthélémy, M. (1995). Des militants de l'école. Les associations de parents d'élèves en France. Revue Française de Sociologie, 26 (3), 439-472.

Castel, R. (2009). La montée des incertitudes. Paris : Seuil.

Dubet, F. (2002). Le déclin de l'institution. Paris : Seuil.

Fablet, D. (2008). L'émergence de la notion de parentalité en milieu(x) professionnels(s). Société et Jeunesse en Difficulté, 5.

Accès en ligne : http://sejed.revues.org/index3532.html

Fassin, D. (2004). Des maux indicibles. Sociologie des lieux d'écoute. Paris : La Découverte.

Foucault, M. (1978). Sécurité, territoire et population. Annuaire du Collège de France. Histoire des systèmes de pensée, année 1977-

\footnotetext{
1 En déclinant ici la notion d' «individu par défaut » proposée par R. Castel, c'est-à-dire incapable d'assumer la charge de la responsabilité et de l'autonomie qui lui est assignée.
} 
1978, 445-449. (repris dans Foucault, M., (2001). Dits et écrits II, 1976-1988 (pp.719-723). Paris : Gallimard.

Gavarini, L. (2001). La passion de l'enfant. Filiation, procréation et éducation à l'aube du XXIème siècle. Paris : Denoël.

Genard, J.-L., \& Cantelli, F. (Eds.) (2007). Action publique et subjectivité. Paris : L.G.D.J. coll. « Droit et société », tome 46.

Glasman, D. (1992). "Parents" ou "familles" : critique d’un vocabulaire générique. Revue Française de Pédagogie, 100, 19-33.

Gombert, P. (2006). Les associations de parents d'élèves en France : approche socio-historique et mutations idéologiques. Revue Française de Pédagogie, 162, 59-66.

Houzel, D. (Ed.) (1999). Les enjeux de la parentalité. Ramonville : Erès. Inspection Générale des Affaires Sociales (IGAS) (2004), Evaluation du dispositif des Réseaux d'écoute, d'appui et d'accompagnement des Parents.

Kherroubi, M. (Ed.) (2008). Des parents dans l'école. Ramonville : Erès. Lamihi, A., \& Monceau, G. (Eds.) (2002). Institution et implication. L'œuvre de René Lourau. Paris : Syllepse.

Le Strat, P.-N. (1997). L'implication, une nouvelle base de l'intervention sociale. Paris : Anthropos.

Missonnier, S. (2004). La relation d'objet virtuel et la parentalité ingénue. Adolescence, 22, (1), 119-131.

Monceau, G. (2003). Pratiques socianalytiques et socio-clinique institutionnelle. L'Homme et la Société, 147-148, 11-33.

Monceau, G. $\left(2008^{\mathrm{a}}\right)$. Implications scolaires des parents et devenirs scolaires des enfants. In M. Kherroubi (Ed.), Des parents dans l'école (pp. 37-87). Ramonville : Érès.

Monceau, G. $\left(2008^{\mathrm{b}}\right)$. Como as instituições permeiam as práticas profissionais. Sócio-clínica institucional e formação de professores (Comment les institutions imprègnent les pratiques professionnelles. Socio-clinique institutionnelle et formation d'enseignants). In S.G. Pimenta \& M.A. Franco Santoro (Eds.), Pesquisa em educação. Vol 1. Possibilidades investigativas/formativas da pesquisa-ação (pp. 27-73). São Paulo : Ed. Loyola (Brésil).

Monceau, G. $\left(2009^{\mathrm{a}}\right)$. L'usage du concept de résistance pour analyser la coopération des parents d'élèves avec les enseignants dans l'institution scolaire. Nouvelle Revue de Psychosociologie, 7, 151165.

Monceau, G. $\left(2009^{b}\right)$. Efficacité des mécanismes de représentation des parents d'élèves : efficacité ou équité ? ", Faculté d'éducation de l'UNISANTOS, Santos (Brésil), Pesquiseduca, 1 (1), 49-56. Accès en ligne :

http://www.diagramaeditorial.com.br/pesquisaeduca/edicao-atual 
Neirinck, C. (2002), Parenté et parentalité. Aspects juridiques. Adolescence, 20 (3), 621-633.

Samson, D. (2005). Les technologies de l'implication : une dérive productiviste d'une double mise en scène dans l'écrit. Éducation permanente, 162, 51-64.

Sellenet, C. (2007). La parentalité décryptée. Pertinence et dérives d'un concept. Paris : L'Harmattan.

Warzée, A. et coll., (2006). La place et le rôle des parents dans l'école (rapport au Ministre de l'Education nationale et de la recherche), octobre 2006.

van Zanten, A. (2001). L'école de la périphérie. Scolarité et ségrégation en banlieue. Paris : PUF. 